\title{
Embarazo ectópico cornual recurrente: caso clínico y revisión de la literatura
}

\author{
Janer Sepúlveda A. ${ }^{1}$, Daniel Torrado A. ${ }^{a}$ \\ 1 Departamento de Ginecología y Obstetricia, Universidad Industrial de Santander. Director del Grupo de Investigación \\ GINO. Bucaramanga, Colombia.
}

a Alumno de Medicina, Universidad Industrial de Santander. Miembro del Grupo de Investigación GINO. Bucaramanga, Colombia.

\section{RESUMEN}

Objetivo: Reportar un caso de embarazo ectópico cornual recurrente, al que se le realizó manejo por laparoscopia. Realizar una revisión de la literatura sobre este tema. Caso clínico: Se presenta el caso de una paciente de 34 años, con embarazo cornual recurrente a quien se le realizó manejo exitoso por laparoscopia. Método: Se realizó una búsqueda bibliográfica en las bases de datos con las palabras clave: pregnancy, intersticial y cornual ectopic en Medline, PubMed, Embase, Cochrane Data base, Ovid, Hinari, Scielo y Bireme. Resultados: La búsqueda inicial arrojó 311 artículos, de los cuales se seleccionaron 116 y posteriormente 74 artículos, que estaban relacionados con el tema a revisar, finalmente se escogieron 38 artículos con texto completo. Conclusión: El embarazo ectópico cornual o intersticial tienen los mismos factores de riesgo que el embarazo tubárico. El avance en ecografía y los niveles de $\beta$ HCG han permitido un diagnóstico precoz, lo que permite realizar tratamientos médico o quirúrgico más conservadores, donde la laparoscopia ha empezado a tener un papel muy importante.

\section{PALABRAS CLAVE: Laparoscopia, embarazo intersticial, embarazo ectópico cornual}

\section{SUMMARY}

Objective: To report a recurrent cornual ectopic pregnancy who underwent laparoscopic management and review the literature on this topic. Case report: 34 year-old patient with a recurrent cornual ectopic pregnancy who underwent successful laparoscopic management. Method: A literature search was performed in databases Medline, PubMed, Embase, Cochrane Data Base, Ovid, Hinari, Scielo and Bireme, with the keywords: pregnancy, interstitial and cornual ectopic pregnancy. Results: The initial search yielded 311 articles of which 116 were selected and then 74 who were related to the subject to review, finally 38 full text articles were selected. Conclusion: Cornual or interstitial ectopic pregnancy has the same risk factors that tubal pregnancy. Advances in ultrasound and $\beta H C G$ levels have allowed earlier diagnosis allowing more conservative treatments either medical or surgical treatment where laparoscopy has begun to play an important role.

KEY WORDS: Laparoscopy, interstitial pregnancy, cornual ectopic pregnancy 


\section{INTRODUCCIÓN}

El embarazo ectópico ocurre cuando el óvulo fecundado se implanta en un sitio diferente a la cavidad uterina normal; cuando nos referimos a cornual o intersticial, es aquel que ocurre en la porción proximal de la tuba uterina, donde se rodea de pared muscular uterina $(1,2)$. Esta porción intersticial mide 7 milímetros $(\mathrm{mm})$ de ancho y 10 a $20 \mathrm{~mm}$ de largo (2).

Algunos autores lo subdividen en angular cuando se implanta medial a la inserción del ligamento redondo, e intersticial al que es lateral a este ligamento $(2,3,4)$, y dejan el término cornual para referirse al embarazo cuando se desarrolla en un cuerno de un útero bicorne $(3,4,5)$. Refieren que hay que distinguir entre estas tres condiciones porque el manejo es diferente; el intersticial es el ectópico con peor pronóstico que requiere siempre tratamiento; el angular se refiere a un embarazo intrauterino viable que se implanta en uno de los ángulos de la cavidad uterina y termina en un aborto involuntario en el $38,5 \%$ de los casos, además dolor pélvico persistente o sangrado vaginal recurrente durante el embarazo, retención de la placenta y ruptura uterina, son complicaciones que se pueden presentar.

En muchos casos los síntomas remiten y puede continuar como un embarazo normal $(3,4)$. En el embarazo cornual la evolución clínica varía según el tamaño y la expansión del cuerno afectado $(3,4)$, sin embargo, en la literatura médica, los términos cornual e intersticial, se utilizan indistintamente y rara vez se menciona el ectópico angular $(6,7,8)$, por lo tanto, en este artículo vamos a utilizar cornual e intersticial como sinónimos.

Un embarazo ectópico tiene una incidencia de $0,2 \%$, siendo la porción ampular la localización más frecuente. La incidencia de la porción cornual es del $3 \%$ del total de ectópicos $(2,8)$, con una tasa de mortalidad materna del embarazo cornual de un 2 a $5 \%$ (9), y causa el $20 \%$ de las muertes por embarazo ectópico $(10,11)$. Por su localización el diagnóstico es difícil, lo que puede llevar a que se presente como complicación ruptura con hemoperitoneo (2). De los embarazos tubáricos es el que tarda más tiempo en romperse, por la capacidad de distensibilidad del miometrio generalmente hasta las 8 semanas, sin embargo, se han reportado rupturas más tempranas $(12,13)$, o lo contrario por el sitio anatómico puede conducir a un retraso en el diagnóstico y la ruptura del útero en el $20 \%$ de los casos con embarazos mayores de 12 semanas (14).

El embarazo cornual también puede ser parte de un embarazo heterotópico que acontece en el $1 \%$ de todos los casos de embarazo ectópico, generalmente asociado a técnicas de fertilización in vitro $(15,16,17)$. El embarazo ectópico cornual es raro, difícil de diagnosticar y es un desafío quirúrgico, donde la laparoscopía juega un papel importante $(12,18)$.

Basados en lo anterior, consideramos que el embarazo ectópico cornual es un tema de interés para los gineco-obstetras, porque debe incluirse entre los diagnósticos diferenciales en mujeres con hemorragia del primer trimestre.

El objetivo de este trabajo es presentar un caso reportado de embarazo ectópico cornual recurrente, al cual se le realizó manejo por laparoscopia.

\section{Caso clínico}

Paciente de 34 años, Gesta 3-Para 0-Abortos 2-Ectópico 1, que consulta por amenorrea de 6 semanas, asintomática. Se le realiza ecografía, que evidencia embarazo ectópico, por lo cual se solicita valoración por cirugía laparoscópica. Como antecedentes de importancia presenta: 1. colecistectomía y manga gástrica por laparoscopia; 2. laparoscopia diagnóstica por embarazo ectópico, de localización cornual y manejo médico con metotrexato; 3 . histeroscopia quirúrgica por síndrome adherencial intrauterino; 4. fumadora de un paquete de cigarrillos al día.

Al examen físico, se encuentra asintomática con útero aumentado de tamaño, compatible con 6 semanas Se realiza nueva ecografía por vía transvaginal, donde se evidencia embarazo ectópico cornual de $3 \mathrm{~cm}$, con con actividad cardiaca positiva (Figura 1), $\beta$ HCG de $15.873 \mathrm{mUl} / \mathrm{ml}$. Se lleva a laparoscopia y se realiza procedimiento sin complicaciones, con egreso el mismo día de la cirugía.

Para la técnica laparoscópica se realizó incisión de $10 \mathrm{~mm}$ infra-umbilical con técnica cerrada con aguja de Veress con presión para la entrada de 20 $\mathrm{mm}$ de mercurio $(\mathrm{HG})$ de presión y posterior se restablece a $15 \mathrm{~mm}$ de $\mathrm{HG}$, se realizan 2 punciones

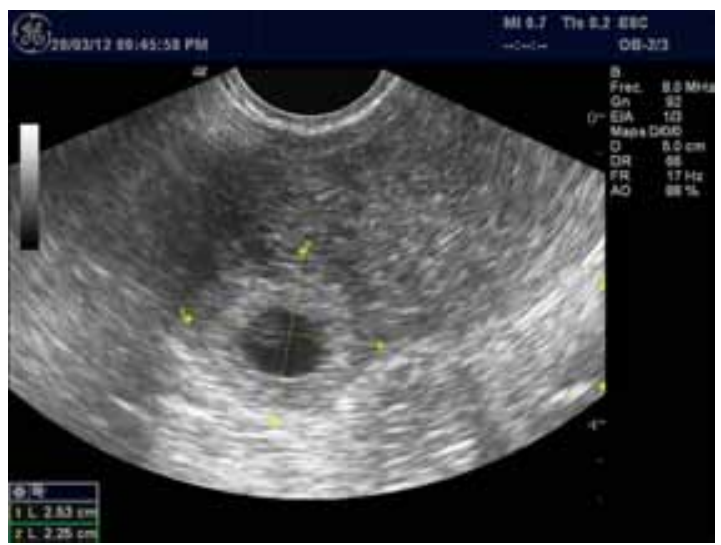

Figura 1. Caso clínico: Ecografía transvaginal que muestra la presencia de embarazo ectópico cornual no complicado. 
de $5 \mathrm{~mm}$ para-rectal izquierda y una a derecha. Se realiza infiltración de 4 unidades de vasopresina diluidas y se realiza una sutura en jareta aproximadamente $5 \mathrm{~mm}$ por debajo del ectópico, se procede a realizar resección del cuerno, con bipolar 25 Watt y tijeras. Duración del procedimiento de 80 minutos y pérdida sanguínea aproximada de $20 \mathrm{ml}$ de sangre.

Posterior a la cirugía se recomienda que debe esperar seis meses para un nuevo embarazo, sin embargo, la paciente acude a los 5 meses con prueba de embarazo positivo, se documenta embarazo intrauterino, el cual se desarrolla sin complicaciones, se realiza cesárea a las 36 semanas, por trabajo de parto pretérmino, se obtiene un recién nacido de sexo femenino en buenas condiciones, con peso adecuado para la edad gestacional. Se revisa zona de ectópico se encuentra pared uterina indemne.

\section{METODOLOGÍA}

Se realizó una búsqueda bibliográfica en las bases de datos con la palabras clave: pregnancy, intersticial y cornual ectopic en Medline, PubMed, Embase, Cochrane Data base, Ovid, Hinari, Scielo y Bireme. Con respecto a los aspectos éticos, se guardó confidencialidad de la información, se realizó consentimiento informado por la paciente para la presentación y publicación del caso clínico.

\section{RESULTADOS}

La búsqueda inicial arrojó 311 artículos, de los cuales se seleccionaron 116 y posteriormente 74 , que estaban relacionados con el tema a revisar, finalmente se escogieron 38 artículos, los cuales se encontraron con texto completo.

Factores de riesgo. El embarazo ectópico cornual tiene los mismos factores de riesgo que los otros tipos de embarazos ectópicos: enfermedad pélvica inflamatoria (EPI), embarazo por reproducción asistida, consumo de cigarrillos, cirugía pélvica previa, embarazo ectópico anterior y el antecedente de salpingectomía ipsilateral $(13,19)$. En una serie de casos, Tulandi y Al-Jaroudi (20), en 32 pacientes encontraron que el factor más importante fue el daño de una trompa en embarazo ectópico previo $(40,6 \%)$, salpingectomía ipsilateral o bilateral $(37,5 \%)$, fertilización in vitro $(34,4 \%)$, historia de enfermedad de transmisión sexual $(25,0 \%)$, e inducción de ovulación $(3,1 \%)$.

Diagnóstico. La clínica incluye la triada clásica de amenorrea, dolor abdominal y hemorragia genital anormal (sobre todo cuando se ha roto) (2), sin embargo, no siempre están presente todos los síntomas. En una serie de casos de $\mathrm{Ng}$ y cols (12), se reportó que de una muestra de 53 casos: el $79,2 \%$ presentó dolor y/o sangrado, $18,8 \%$ se presentó asintomática y 1,8\% con shock hipovolé- mico; mientras que Soriano y cols (13), informan 20 casos donde se presentaron con dolor abdominal en $52 \%$, sangrado genital en $29,6 \%$, asintomáticos en $33 \%$ y shock hipovolémico en $22 \%$ de los casos (13).

El diagnóstico es clínico junto con el hallazgo ecográfico y un aumento de los niveles de $\beta H C G$ $(2,18)$. En la serie de casos de $\mathrm{Ng}$ y cols $(12)$, reportaron diagnóstico en $65,6 \%$ mediante ecografía, el resto se realizó por laparoscopia, por diagnóstico dudoso. El estudio ecográfico suele mostrar un útero vacío con un saco gestacional localizado a nivel cornual, con una delgada capa de músculo liso generalmente mayor de $5 \mathrm{~mm}$ del útero, a menos de $1 \mathrm{~cm}$ desde el borde lateral de la cavidad uterina (21). También existe el hallazgo del signo de la línea intersticial con $80 \%$ de sensibilidad y $98 \%$ de especificidad, viéndose una línea ecogénica que bordea el saco gestacional intramural (2). Por otro lado, hay que tener en cuenta dentro de diagnóstico diferencial, el aborto retenido, útero septado y útero didelfo (18).

Tratamiento no quirúrgico. El tratamiento no quirúrgico se realiza básicamente con metotrexato $(3,6,22,23)$, las ventajas que ofrece es evitar la cicatriz en el útero y los riesgos asociados a la cirugía (3), sin embargo, este tratamiento también ofrece riesgos como subsecuente ruptura del cuerno, lo que conlleva a riesgo de muerte por hemorragia (3).

Tang y cols (23), en 11 pacientes usaron metotrexato endovenoso con dosis inicial de $100 \mathrm{mg}$ y posteriormente $200 \mathrm{mg}$, combinado con ácido folínico; una paciente requirió laparotomía por ruptura del ectópico, los niveles de $\beta$ HCG no fueron tenidos en cuenta. Por otro lado, Jermy y cols (21), realizaron un estudio prospectivo con metotrexato intramuscular $50 \mathrm{mg}$ por metro cuadrado y una segunda dosis si no hay disminución de los niveles de $\beta \mathrm{HCG}$ en un $15 \%$ en el día séptimo comparado con los niveles del cuarto día; todos los casos fueron tratados con éxito con una dosis de metotrexato, con valores menores de $\beta H C G$ de $5000 \mathrm{mU} / \mathrm{ml}$, los valores mayores a este nivel requirieron una segunda dosis, sin embargo, se presentaron dos casos excepcionales con valores iniciales de 31.381 $\mathrm{mU} / \mathrm{ml}$ y $9.684 \mathrm{mU} / \mathrm{ml}$, que también respondieron a una sola dosis (21). Sagiv y cols (24), reportan tres pacientes manejadas con inyección local de metotrexato, usando laparoscopia, con buena respuesta.

También se ha usado el cloruro de potasio y etopósido en inyección local $(2,3)$, pero el riesgo de embarazo intersticial recurrente o ruptura uterina en un nuevo embarazo permanece incierto (3).

Tratamiento quirúrgico. El tratamiento tradicional ha sido la histerectomía o resección cornual por laparotomía $(3,6,10,11,13,14,19,22-24,26-29,31,32)$, sin embargo, con el advenimiento de la ecografía y 
la $\beta C G(3,11,19)$ se realizan diagnósticos más tempranos y ha permitido escoger nuevas alternativas menos agresivas para el tratamiento.

El manejo quirúrgico de elección actual es la vía laparoscópica $(12,13,18,19,26)$. Las técnicas implicadas son: salpingectomía, resección en cuña y la cuernostomía, aunque esta última se ha asociado a más recurrencia de embarazo ectópico. La elección del procedimiento depende del tamaño y del deseo de conservar la fertilidad para un futuro embarazo, donde el uso de la sutura es la piedra angular para lograr la hemostasia $(12,18,19)$. Se prefiere el manejo por esta vía, porque el tiempo de estancia hospitalaria y la pérdida sanguínea es menor comparado con la laparotomía, un retorno a las actividades normales más rápida (3) e incluso se ha reportado manejo por un solo puerto como en el reporte de Lee y cols (1). Por otro lado los principales retos en la cirugía por el embarazo ectópico cornual, son la hemostasia y la actividad trofoblástica persistente después de la cirugía, preservar la permeabilidad tubárica y la integridad cornual (18).

En una serie de casos de $\mathrm{Ng}$ y cols (12), reportaron 53 casos, de los cuales 33 fueron manejados con resección en cuña laparoscópica, en embarazo mayor de $3 \mathrm{~cm} ; 13$ de los casos con cuernostomía laparoscópica para embarazos de 1 a $2 \mathrm{~cm}$ y 7 casos con salpingectomía laparoscópica; en cuanto a la fertilidad 10 de los 53 casos pudieron quedar embarazadas, sin presentar ruptura uterina en el proceso gestacional. Otros autores recomiendan para tamaños menores de $3,5 \mathrm{~cm}$ de diámetro cuernostomía, y para tamaños mayores de $4 \mathrm{~cm}$ resección cornual $(2,18,33)$.

Tinelli y cols (2), reportan 3 casos de embarazo ectópico cornual con un tamaño entre 2,5 a 2,9 cm de diámetro, un caso de ellos con hemoperitoneo; todos fueron manejados por laparoscopia y metotrexato intramuscular postquirúrgico sin complicaciones. Igualmente, Moon y cols (9), reportaron una serie de 24 casos, donde utilizan vasopresina y energía, sutura circular o en jareta alrededor del ectópico cornual o el uso de endolop, y llegan a la conclusión que estas dos últimas son seguras y efectivas para controlar la pérdida sanguínea.

En algunos casos se necesita tratamiento postquirúrgico, complementaria con metotrexato, dosis de $50 \mathrm{mg}$ por metro cuadrado, para completar la finalización del embarazo, generalmente cuando la disminución de $\beta \mathrm{HCG}$ postquirúrgica fue menos del $15 \%$ respecto a los valores previos $(12,13,18)$.

Embolización selectiva de la arteria uterina. La embolización selectiva de la arteria uterina continúa siendo experimental, cuando es utilizada en forma aislada (3), pero se ha usado en combinación con metotrexato con buenos resultados (25). Takeda y cols (27), reportan un caso usando la embolización de la arteria uterina en forma preoperatoria y posterior laparoscopia, completando finalmente con metotrexato local con tratamiento exitoso (27).

Evacuación transcervical. Thakur y cols (34), reportaron 4 casos realizando una combinación de laparoscopia y guía de ultrasonido, dilatación del canal cervical y posterior dilatación del ostium tubárico con succión y evacuación (34); no hay reporte sobre efecto de recurrencia y daño del cuerpo uterino para fertilidad futura (3). Oliver y cols (35), también reportaron 5 casos con éxito. Katz y cols (4), reportan en dos pacientes un manejo combinado de laparoscopia e histeroscopia, con posterior aspiración del tejido trofoblástico. Ross y cols (8), describen dos casos a quienes les realiza primero laparoscopia y posteriormente bajo visión ecográfica se les realiza dilatación y curetaje, con nueva revisión laparoscópica, completan con una dosis de metotrexato (8).

Histeroscopia. La histeroscopia también ha sido reportada para el tratamiento del embarazo ectópico cornual $(20,29)$. Sanz y cols $(36)$, reportan el uso de la histeroscopia para romper un saco persistente después del tratamiento con metotrexato y realizan bajo visión ecográfica la extracción del tejido placentario. También ha sido reportado en combinación con laparoscopia, como se describió anteriormente (4).

Embarazo futuro. En embarazo ectópico cornual en mujeres interesadas en fertilidad futura los procedimientos de elección deberían ser la salpingotomía y la resección de la región del cuerno uterino por vía laparoscópica $(2,33)$. En cuanto a la vía de parto del próximo embarazo, muchos cirujanos tienden a recomendar cesárea para disminuir el conocido riesgo de rotura uterina $(2,37)$.

Recurrencia del embarazo cornual. El embarazo intersticial recurrente y la incidencia de ocurrencia es desconocida (17), puede presentarse tan pronto como a los 4 meses y tan tarde como en 5 años después del primer embarazo ectópico cornual, parece no haber ninguna correlación entre el método terapéutico usado en el primer embarazo ectópico y la recurrencia (26), esta incluso puede darse ante cirugías con buen resultado anatómico (12). En la Tabla I se describen los pocos casos registrados de ectópico recurrente $(17,24,26,32,38)$, también se anexa el caso que reportamos en este artículo. 
Tabla I

COMPARACIÓN DE CASOS EMBARAZO ECTÓPICO CORNUAL RECURRENTE

\begin{tabular}{|c|c|c|c|c|c|}
\hline No. & Autor & Año & $\begin{array}{l}\text { Tratamiento en el primer embarazo } \\
\text { cornual }\end{array}$ & $\begin{array}{l}\text { Tiempo de } \\
\text { recurrencia } \\
\text { (meses) }\end{array}$ & $\begin{array}{l}\text { Tratamiento en el } \\
\text { embarazo cornual } \\
\text { recurrente }\end{array}$ \\
\hline 1 & Petersen \& Clausen & 1989 & Laparotomía con cuernostomía & 12 & $\begin{array}{l}\text { Laparotomía con } \\
\text { cuernostomía }\end{array}$ \\
\hline 2 & Wittich & 1998 & Laparotomía con resección en cuña & 16 & $\begin{array}{l}\text { Laparotomía con } \\
\text { resección en cuña }\end{array}$ \\
\hline 3 & Sungurtekin \& Uyar & 1998 & MTX sistémico & 17 & $\begin{array}{l}\text { Laparotomía con } \\
\text { resección en cuña }\end{array}$ \\
\hline 4 & Vilos & 2001 & Salpingectomía por laparoscopia & 10 & $\begin{array}{l}\text { Salpingectomía por } \\
\text { laparoscopia }\end{array}$ \\
\hline 5 & Sagiv et al & 2001 & $\begin{array}{l}\text { Laparoscopia e inyección local de } \\
\text { MTX }\end{array}$ & 6 & $\begin{array}{l}\text { Cuernostomía por } \\
\text { Laparoscopia }\end{array}$ \\
\hline 6 & Sharma \& Aziz & 2004 & Laparotomía con resección en cuña & 72 & Histerectomia subtotal \\
\hline 7 & $\begin{array}{l}\text { Van der Weiden \& } \\
\text { Karsdorp }\end{array}$ & 2005 & Feticidio selectivo de heterotópico & 24 & MTX sistémico \\
\hline 8 & Faleyimu et al & 2008 & Laparotomía con resección en cuña & 60 & $\begin{array}{l}\text { Laparotomía con } \\
\text { salpingo-ooforectomía }\end{array}$ \\
\hline 9 & Sahoo et al & 2009 & $\begin{array}{l}\text { Endoloop por laparoscopia con } \\
\text { MTX sistémico postoperatorio, uso } \\
\text { de mifeprestone }\end{array}$ & 12 & $\begin{array}{l}\text { Endoloop por laparos- } \\
\text { copia }\end{array}$ \\
\hline \multirow[t]{2}{*}{10} & Faraj \& Steel & 2009 & MTX sistémico & 8 & MTX sistémico \\
\hline & Siow \& Ng & 2011 & & & \\
\hline 11 & Caso I & & $\begin{array}{l}\text { Cuernostomía derecha por lapa- } \\
\text { roscopia }\end{array}$ & 18 & $\begin{array}{l}\text { Resección del cuerno } \\
\text { derecho por laparos- } \\
\text { copia }\end{array}$ \\
\hline 12 & Caso 2 & & $\begin{array}{l}\text { Cuernostomía derecha por laparos- } \\
\text { copia y MTX sistémico }\end{array}$ & 5 & $\begin{array}{l}\text { Resección del cuerno } \\
\text { derecho por laparos- } \\
\text { copia }\end{array}$ \\
\hline 13 & Caso 3 & & $\begin{array}{l}\text { Cuernostomía izquierdo por lapa- } \\
\text { roscopia }\end{array}$ & 26 & $\begin{array}{l}\text { Resección del cuerno } \\
\text { izquierdo por laparos- } \\
\text { copia }\end{array}$ \\
\hline \multirow[t]{2}{*}{14} & Caso 4 & & $\begin{array}{l}\text { Resección del cuerno izquierdo por } \\
\text { laparoscopia y MTX sistémico }\end{array}$ & 4 & $\begin{array}{l}\text { Resección del cuerno } \\
\text { derecho por laparos- } \\
\text { copia y salpingecto- } \\
\text { mía }\end{array}$ \\
\hline & & & & 32 & $\begin{array}{l}\text { Resección del cuerno } \\
\text { derecho por laparos- } \\
\text { copia }\end{array}$ \\
\hline 15 & Caso presentado & 2015 & MTX & 72 & $\begin{array}{l}\text { Resección del cuerno } \\
\text { izquierdo por laparos- } \\
\text { copia }\end{array}$ \\
\hline
\end{tabular}

MTX: Metotrexato. 


\section{CONCLUSIONES}

El embarazo ectópico cornual o intersticial tienen los mismos factores de riesgo que el embarazo tubárico. La ecografía y los niveles de BHCG, han permitido un diagnóstico más temprano, lo que conlleva a realizar tratamientos más conservadores, ya sea médico o quirúrgico, donde la laparoscopia ha empezado a tener un papel muy importante.

\section{REFERENCIAS}

1. Lee ES, Hahn HS, Park BJ, Ro DY, Kim JH, Kim Y. Single-port laparoscopic cornual resection for a spontaneous cornual ectopic pregnancy following ipsilateral salpingectomy. Fertil Steril 2011;96(2):106-10.

2. Tinelli $A$, Malvasi A, Pellegrino M, Pontrelli G, Martulli $\mathrm{B}$, Tsin DA. Laparoscopical management of cornual pregnancies: a report of three cases. Eur J Obstet Gynaecol Reprod Biol 2010;151:199-202

3. Moawad NS, Mahajan ST, Moniz MH, Taylor SE, Hurd WW. Current diagnosis and treatment of interstitial pregnancy. Am J Obstet Gynecol 2010;202:15-29.

4. Katz DL, Barrett JP, Sanfilippo JS, Badway DM. Combined hysteroscopy and laparoscopy in the treatment of interstitial pregnancy. Am J Obstet Gynecol 2003;188:113-4.

5. Szylit NA, Podgaec S, Traina E, Oliveira Rde C. Video laparoscopic intervention for an interstitial pregnancy after failure of clinical treatment. Sao Paulo Med J 2012;130(3):202-7.

6. Verity L, Ludlow J, Dickinson JE. Interstitial ectopic pregnancy: a contemporary case series. Aust N Z J Obstet Gynaecol 2003;43:232-5.

7. Günenç Z, Bingöl B, Çelik A, Bozkurt S, Özekici U. Laparoscopic surgery of interstitial (cornual) pregnancy, a case report. J Turkish-German Gynecol Assoc 2010;11:102-4.

8. Ross R, Lindheim SR, Olive DL, Pritts EA. Cornual gestation: a systematic literature review and two case reports of a novel treatment regimen. J Minim Invasive Gynecol 2006;13(1):74-8.

9. Moon HS, Choi YJ, Park YH, Kim SG. New endoscopic operations for interstitial pregnancies. Am J Obstet Gynecol 2000;182(1(Part 1)):114-21.

10. Jaeger $C$, Reich A, Kreienberg R, Flock F. Suitable laparoscopic surgery in the treatment of ectopic interstitial pregnancy. Gynecol Surg 2006;3:1-5.

11. Gezer A, Mutlu H. Laparoscopic management of cornual pregnancy without sutures. Arch Gynecol Obstet 2004; 270:194-6.

12. Ng S, Hamontri S, Chua I, Chern B, Siow A. Laparoscopic management of 53 cases of corneal ectopic pregnancy. Fertil Steril 2009;92(2):448-52.

13. Soriano D, Vicus D, Mashiach R, Schiff E, Seidman D, Goldenberg M. Laparoscopic treatment of cornual pregnancy: a series of 20 consecutive cases. Fertil Steril 2008; 90(3): 839-43.

14. Pluchino N,Ninni F, Angioni S, Carmignani A, Genazzani AR, Cela V. Spontaneous cornual pregnancy after homolateral salpingectomy for an earlier tubal pregnancy: a case report and literature review. J Minim Invasive Gynecol 2009;16(2):208-11.
15. Pasic RP, Hammons G, Gardner JS, Hainer M. Laparoscopic treatment of cornual heterotopic pregnancy. J Am Assoc Gynecol Laparosc 2002;9(3):372-5.

16. Ćorić M, Barišić D, Sterlec M. Laparoscopic approach to interstitial pregnancy. Arch Gynecol Obstet 2004;270:287-9.

17. Van der Weiden RMF, Karsdorp VHM. Recurrent cornual pregnancy after heterotopic cornual pregnancy successfully treated with systemic methotrexate. Arch Gynecol Obstet 2005;273:180-1.

18. MacRae R, Olowu O, Rizzuto MI, Odejinmi F. Diagnosis and laparoscopic management of 11 consecutive cases of cornual ectopic pregnancy. Arch Gynecol Obstet 2009;280:59-64.

19. Choi YS, Eun DS, Choi J, Shin KS, Choi JH, Park HD. Laparoscopic cornuotomy using a temporary tourniquet suture and diluted vasopressin injection in interstitial pregnancy. Fertil Steril 2009;91(5):1933-7.

20. Tulandi T, Al-Jaroudi D. Interstitial pregnancy: results generated from the Society of Reproductive Surgeons registry. Obstet Gynecol 2004;103:47-50.

21. Jermy K, Thomas J, Doo A, Bourne T. The conservative management of interstitial pregnancy. BJOG 2004;111:1283-8.

22. Lau S, Tulandi T. Conservative medical and surgical management of interstitial ectopic pregnancy. Fertil Steril 1999;72:207-15.

23. Tang A, Baartz D, Khoo SK. A medical management of interstitial ectopic pregnancy: A 5-year clinical study. Aust N Z J Obstet Gynaecol 2006; 46:107-11.

24. Sagiv R, Golan A, Arbel-Alon S, Glezerman M. Three conservative approaches to treatment of interstitial pregnancy. J Am Assoc Gynecol Laparosc 2001;8(1):154-8.

25. Deruelle P, Lucot JP, Lions C, Robert Y. Management of interstitial pregnancy using selective uterine artery embolization. Obstet Gynecol 2005;106(5 Pt 2):11657.

26. Siow A, Ng S. Laparoscopic Management of 4 Cases of Recurrent Cornual Ectopic Pregnancy and Review of Literature. JMIG 2011;18(3):296-302.

27. Takeda A, Koyama K, Imoto S, Mori M, Sakai K, Nakamura $H$. Successful management of interstitial pregnancy with fetal cardiac activity by laparoscopicassisted cornual resection with preoperative transcatheter uterine artery embolization. Arch Gynecol Obstet 2009;280(2):305-8.

28. Bouyer J, Coste J, Fernandez H, Pouly JL, JobSpira N. Sites of ectopic pregnancy: a 10-year population-based study of 1800 cases. Hum Reprod 2002;17:3224-30.

29. Buxant F, Ansion MH, Noel JC, Anaf V, Simon P. Laparoscopic management of a cornual ectopic pregnancy. Gynecol Surg 2005; 2:197-200.

30. Cortés A, García E, Audifred J ,González PA. Embarazo cornual. Manejo laparoscópico y presentación de un caso. Ginecol Obstet Méx 1999;67(7): 300-1.

31. Bremner T, Cela V, Luciano A, Surgical management of interstitial pregnancy. J Am Assoc Gynecol Laparosc 2000;7(3):387-9.

32. Vilos GA. Laparoscopic ligation and resection of two ipsilateral interstitial pregnancies in the same patient. J Am Assoc Gynecol Laparosc 2001;8(2):299-302.

33. Zarhi J, Campaña C, Brito R, Stuardo. P, Schalper J. 
Manejo laparoscópico conservador de embarazo cornual. Rev Chil Obstet Ginecol 2003;68(1):36-41.

34. Thakur Y, Coker A, Morris J, Oliver R. Laparoscopic and ultrasound-guided transcervical evacuation of cornual ectopic pregnancy: an alternative approach. J Obstet Gynaecoly 2004;24(7):809-10.

35. Oliver R, Malik M, Coker A, Morris J. Management of extra-tubal and rare ectopic pregnancies: case series and review of current literature. Arch Gynecol Obstet 2007;276:125-31.
36. Sanz LE, Verosko J. Hysteroscopic management of cornual ectopic pregnancy. Obstet Gynecol 2002;99:941-4.

37. Sungurtekin $U$, Uyar $Y$. Recurrent interstitial pregnancy. Aust N Z J Obstet Gynaecol 1998;38(4):438-40.

38. Arellano A, Antillón J, González AJ, Mojarra JM. Manejo conservador laparoscópico de un embarazo ectópico intersticial no roto. Reporte de caso, descripción de la técnica quirúrgica y revisión de la literatura Perinatol Reprod Hum 2012;26 (2):121-8. 